\title{
Avaliação por métodos in vitro e in vivo da biodisponibilidade de sulfato ferroso microencapsulado
}

\author{
In vitro and in vivo evaluation of iron bioavailability \\ from microencapsulated ferrous sulfate
}

\author{
Maria Lucia COCATO ${ }^{1}$ \\ Maria Inês RÉ \\ Messias Alves TRINDADE NETO3 \\ Helena Pontes CHIEBAO ${ }^{1}$ \\ Célia COLLI ${ }^{1}$
}

RES U M O

\section{Objetivo}

Avaliar, por métodos in vitro e in vivo, a biodisponibilidade de uma nova forma de sulfato ferroso microencapsulado (genericamente denominado Ferlim), desenvolvido para a fortificação de alimentos, comparando-a com a de ferro eletrolítico $\left(\mathrm{Fe}^{0}\right)$.

\section{Métodos}

A avaliação da dialisabilidade in vitro utilizou como matriz leite em pó reconstituído. A avaliação in vivo, utilizando o método de recuperação de hemoglobina em leitões anêmicos, teve duração de 13 dias e os animais $(\mathrm{n}=23)$ foram agrupados de acordo com o produto do peso $(\mathrm{kg}) \times$ hemoglobina $(\mathrm{g} / \mathrm{dL})$. Como controle foi utilizado $\mathrm{FeSO}_{4} \cdot 7 \mathrm{H}_{2} \mathrm{O}$.

\section{Resultados}

As porcentagens médias de ferro dialisado foram 2,2 (desvio-padrão=0,1)\%, 3,4 (desvio-padrão=0,1)\% e $3,6$ (desvio-padrão $=0,0) \%$ para $\mathrm{FeSO}_{4} \cdot 7 \mathrm{H}_{2} \mathrm{O}$, Ferlim e Fe ${ }^{0}$, respectivamente $(p<0,05)$. A absorção de ferro foi de 16,0 (desvio-padrão $=3,1$ ) \% para o grupo controle, de 15,1 (desvio-padrão=3,8)\% para o grupo Ferlim e de $12,8$ (desvio-padrão $=4,3) \%$ para o grupo $\mathrm{Fe}^{0}$, não sendo significantemente diferentes $(p>0,05)$. As porcentagens de absorção do valor biológico relativo do $\mathrm{FeSO}_{4} \cdot 7 \mathrm{H}_{2} \mathrm{O}$ foram de 94,2 (desvio-padrão=23,8)\% para o grupo Ferlim e de 79,7 (desvio-padrão=26,6)\% para o grupo $\mathrm{Fe}^{0}$, sem diferenças significantes $(p>0,05)$. Em valores numéricos $(p>0,05)$, o grupo $\mathrm{Fe}^{0}$ apresentou menor média de absorção (\%) valor biológico relativo de concentração de ferro total, de ferro heme e não-heme no fígado.

1 Universidade de São Paulo, Faculdade de Ciências Farmacêuticas, Departamento de Alimentos e Nutrição Experimental, Laboratório de Metabolismo e Nutrição Mineral. Av. Prof. Lineu Prestes, 580, Bloco B, Cidade Universitária, 05508-900, São Paulo, SP, Brasil. Correspondência para/Correspondence to: C. COLLI.

2 Instituto de Pesquisas Tecnológicas do Estado de São Paulo, Centro de Tecnologia de Processos e Produtos. São Paulo, SP, Brasil.

3 Universidade de São Paulo, Faculdade de Medicina Veterinária e Zootecnia, Departamento de Nutrição e Produção Animal. Pirassununga, SP, Brasil. 


\section{Conclusão}

A microencapsulação do sulfato ferroso com alginato mantém sua biodisponibilidade, caracterizando-se como boa alternativa para a fortificação de misturas sólidas.

Termos de indexação: biodisponibilidade; composição de medicamentos; ferro.

\section{A B S T R A C T}

\section{Objective}

To evaluate, by in vitro and in vivo methods, the bioavailability of a new microencapsulated ferrous sulfate (Ferlim) developed for food fortification, and compare it with electrolytic iron $\left(\mathrm{Fe}^{\mathrm{O}}\right)$.

\section{Methods}

In vitro dialyzability assessment used reconstituted milk powder as matrix. In vivo assessment using the hemoglobin regeneration method in anemic piglets lasted for 13 days and the animals $(n=23)$ were grouped according to the product of weight $(\mathrm{kg}) \times$ hemoglobin $(\mathrm{g} / \mathrm{dL})$. $\mathrm{FeSO}_{4} \cdot \mathrm{7H}_{2} \mathrm{O}$ was used as control.

\section{Results}

The percentages of dialyzed iron were 2.2 (standard deviation=0.1)\%, 3.4 (standard deviation=0.1)\% and 3.6 (standard deviation $=0.0) \%$ for $\mathrm{FeSO}_{4} \cdot 7 \mathrm{H}_{2} \mathrm{O}$, Ferlim and $\mathrm{Fe}^{\mathrm{O}}$ respectively $(\mathrm{p}<0.05)$. Iron absorption was 16.0 (standard deviation=3.1)\% for the control group, 15.1 (standard deviation=3.8)\% for the Ferlim group and 12.8 (standard deviation=4.3)\% for the $\mathrm{Fe}^{0}$ group; the differences were not significant ( $\left.p>0.05\right)$. The absorption percentages of the relative biological value of $\mathrm{FeSO}_{4} \cdot \mathrm{7H}_{2} \mathrm{O}$ were 94.2 (standard deviation=23.8)\% for the Ferlim group and 79.7 (standard deviation=26.6)\% for the $\mathrm{Fe}^{0}$ group; the differences were not significant $(p>0.05)$. In numerical values ( $p>0.05)$, the $\mathrm{Fe}^{0}$ group presented the lowest mean relative biological value absorption (\%) and concentration of total iron, heme iron and non-heme iron in the liver.

\section{Conclusion}

Microencapsulation of ferrous sulfate with alginate retains its bioavailability therefore it is a good alternative for the fortification of solid mixtures.

Indexing terms: biological availability; drug compounding; iron.

\section{N T R O D U Ç Ã O}

No Brasil, a Agência Nacional de Vigilância Sanitária, por meio da Resolução RDC 344, de 13 de dezembro de 2002, tornou obrigatória a fortificação das farinhas de trigo e de milho com ferro e ácido fólico ${ }^{1}$, medida para combater a anemia por deficiência de ferro. Estudos conduzidos em crianças nos últimos 20 anos mostram que a prevalência de anemia em alguns estados brasileiros alcança índice de 70\% na faixa etária de 6 a 12 meses de idade ${ }^{2-5}$.

A fortificação de alimentos com sais de ferro, uma das estratégias propostas pela Organização Mundial de Saúde no combate à anemia por deficiência deste mineral, tem como principais vantagens alcançar todos o segmentos da população e não requerer a cooperação do indiví- duo ${ }^{6-8}$. Essa fortificação, entretanto, é mais difícil do que com outros micronutrientes, porque as formas de ferro com boa solubilidade, como $\mathrm{FeSO}_{4}$, são quimicamente reativas e provocam alterações organolépticas indesejáveis quando adicionadas aos alimentos ${ }^{7}$. Por outro lado, compostos de ferro inerte ou sais de ferro insolúveis, como ferro reduzido e fosfato ferroso, não produzem tais alterações, mas têm baixa absorção ${ }^{7,9-11}$.

Dessa forma, a escolha do melhor composto de ferro a ser adicionado ao alimento é um passo essencial no programa de fortificação. Como principais características, o composto de ferro a ser utilizado deve possuir solubilidade elevada e, assim, boa biodisponibilidade, não diminuir o valor nutricional do veículo no qual foi adicionado e não alterar suas propriedades organolépticas ${ }^{12,13}$. Um composto com todas essas características era 
desconhecido até pouco tempo, porém com o advento do processo de microencapsulação de $\mathrm{FeSO}_{4}$, essa possibilidade tornou-se promissora. $\mathrm{Na}$ Argentina, um composto de ferro microencapsulado com lecitina de soja, SFE $141^{\oplus}$, demonstrou elevada biodisponibilidade, tanto em testes in vitro como in vivo ${ }^{14-17}$

O propósito deste estudo foi avaliar a biodisponibilidade de um novo composto de ferro revestido de uma camada de polissacarídeo natural, genericamente denominado Ferlim, desenvolvido no Brasil pelo Instituto de Pesquisas Tecnológicas do Governo do Estado de São Paulo (IPT) ${ }^{18,19}$, para ser utilizado na fortificação de farinha de trigo ${ }^{20}$.

Uma série de caracterizações físico-químicas mostrou que esse novo produto Ferlim, constituído de um núcleo sulfato ferroso revestido de uma camada de polissacarídeo natural extraído de algas marinhas, apresentou características físicas, como granulometria e densidade aparente, próximas às da farinha de trigo, requisitos importantes para a boa mistura e não segregação após a fortificação da mesma ${ }^{20}$. Testes de dissolução em pH 4,5 no meio acetato de sódio também demonstraram que o composto possuía elevada solubilidade ${ }^{21}$, o que é importante para não modificar a boa biodisponibilidade do $\mathrm{FeSO}_{4}$.

Neste trabalho, o objetivo foi aprofundar a caracterização do composto Ferlim, determinando sua biodisponibilidade pela dialisabilidade in vitro e pela recuperação de hemoglobina em leitões anêmicos, comparando-o com o sulfato ferroso não encapsulado e com o $\mathrm{Fe}^{0}$. A utilização de leitões no ensaio in vivo tem como vantagem oferecer condições fisiológicas muito próximas às encontradas em humanos. Suínos e seres humanos são onívoros e seu trato gastrintestinal tem similaridades anatômicas e fisiológicas ${ }^{22}$, como é o caso do pH gástrico e da atividade das enzimas digestivas, principalmente no primeiro estágio de vida 23,24 .

Os resultados da avaliação in vitro permitem o exame pontual de um alimento ou dieta. Já a avaliação in vivo representa um estudo ao longo do tempo, em um grupo anêmico, de modo que elas se complementam.

\section{MÉ T O D OS}

Os compostos de ferro utilizados foram ferro microencapsulado, denominado genericamente Ferlim, preparado pelo Laboratório de Tecnologia de Partículas do Instituto de Pesquisas Tecnológicas do Estado de São Paulo (IPT) ${ }^{18,19}$, sulfato ferroso heptahidratado e $\mathrm{Fe}^{0}$, ambos fornecidos pela empresa Fermavi Eletroquímica $\mathrm{S} / \mathrm{A}$. O Fe ${ }^{0}$ utilizado caracteriza-se pela seguinte granulometria: 0-10 $\mu \mathrm{m}(20 \%), 11-20 \mu \mathrm{m}(30 \%)$ e $21-40 \mu \mathrm{m}(40 \%)$.

\section{Ensaio biológico}

Dieta: A ração de depleção para suínos em crescimento, à base de milho e leite em pó desnatado, foi preparada segundo a National Research Council25 (Tabela 1). Por se tratar de ração à qual não foi adicionada a fonte de ferro, os ingredientes foram previamente analisados para determinar o conteúdo em ferro e evitar o uso de matérias-primas contaminadas com o mineral. Foram formuladas 3 rações de repleção, tendo como base a mesma ração utilizada no período de depleção. O grupo-padrão teve $\mathrm{FeSO}_{4} \cdot 7 \mathrm{H}_{2} \mathrm{O}$ como fonte de ferro e, para os grupos experimentais, as fontes de ferro foram Ferlim (Grupo Ferlim) e ferro eletrolítico (Grupo $\left.\mathrm{Fe}^{0}\right)$. As rações dos grupos experimentais foram formuladas para conter 150mg Fe/kg (Tabela 2) e continham 17\% de proteína e $3 \%$ de gordura em base úmida.

Animais: Foram utilizados 37 leitões de linhagem geneticamente definida, machos castrados e fêmeas, recém-desmamados, mestiços, com 21 dias de idade, pesando, em média, 7,2, $D P=1,4 \mathrm{~kg}$, provenientes da Granja Kager da cidade de Holambra, SP. O experimento foi conduzido no Instituto de Zootecnia de Nova Odessa - APTA -SAA-SP. Ao nascimento, os leitões receberam injeção intramuscular de ferro-dextrano (60mg ao invés dos 200mg normalmente aplicados em leitões recém-nascidos). Na primeira fase do ensaio (fase de depleção), os leitões foram alojados três a três em gaiolas de aço inoxidável, 
sob temperatura controlada $\left(\sim 25^{\circ} \mathrm{C}\right)$, e tiveram livre acesso a ração sem fonte de ferro e água. No sétimo dia, coletou-se o sangue dos animais para a determinação da hemoglobina. Uma vez constatada a anemia [concentração média de hemoglobina de $6,6(1,1)$ g.L.-1], iniciou-se a segunda fase do ensaio (fase de repleção). O protocolo experimental foi aprovado pela Comissão de Ética em Experimentação Animal da Faculdade de Ciências Farmacêuticas da Universidade de São Paulo, SP - em concordância com os princípios em pesquisa animal adotado pelo Colégio Brasileiro de Experimentação Animal (COBEA).

Tabela 1. Composição centesimal das rações de depleção e repleção ${ }^{25}$.

\begin{tabular}{|c|c|}
\hline Ingredientes & $\%$ \\
\hline Milho & 58,11 \\
\hline Leite em pó desnatado & 40,10 \\
\hline Cloreto de colina & 0,08 \\
\hline Fosfato bicálcico & 0,49 \\
\hline Carbonato de cálcio & 0,42 \\
\hline Sal & 0,20 \\
\hline L-triptofano & 0,02 \\
\hline L-lisina & 0,07 \\
\hline Pré-Mix vitamínico* & 0,50 \\
\hline \multicolumn{2}{|l|}{ Minerais** (g) } \\
\hline $\mathrm{ZnO}$ & 13,70 \\
\hline $\mathrm{CuSO}_{4} \cdot 5 \mathrm{H}_{2} \mathrm{O}$ & 2,40 \\
\hline $\mathrm{MNSO}_{4} \cdot \mathrm{H}_{2} \mathrm{O}$ & 1,20 \\
\hline \multicolumn{2}{|l|}{ Composição calculada } \\
\hline Energia metabolizável (kcal/kg) & 3382,00 \\
\hline Proteína bruta (\%) & 18,13 \\
\hline Cálcio (\%) & 0,80 \\
\hline Fósforo total (\%) & 0,65 \\
\hline Fósforo disponível (\%) & 0,51 \\
\hline Lisina digestível (\%) & 1,19 \\
\hline Metionina+cistina digestível (\%) & 0,73 \\
\hline Triptofano digestível (\%) & 0,22 \\
\hline Treonina digestível (\%) & 0,76 \\
\hline
\end{tabular}

* Pré-Mix vitamínico (Composição/kg do produto): Vitamina $\mathrm{A}$ (2.000.000UI); Vitamina $D_{3}(500.000 \mathrm{UI})$; Vitamina $\mathrm{E}(2.500 \mathrm{mg})$; Vitamina $\mathrm{K}_{3}(125 \mathrm{mg})$; Viamina $\mathrm{B}_{1}(375 \mathrm{mg})$; Vitamina $\mathrm{B}_{2}(1.250 \mathrm{mg})$; Vitamina $B_{6}(500 \mathrm{mg})$; Vitamina $B_{12}(5.000 \mathrm{mg})$; Niacina $(6.250 \mathrm{mg})$; **Ferro: Grupo-padrão: adição de $\mathrm{FeSO}_{4} \cdot 7 \mathrm{H}_{2} \mathrm{O}$; Grupos experimentais: Grupo Ferlim: adição de Ferlim e Grupo $\mathrm{Fe}^{0}$ : adição de ferro eletrolítico. Todas as rações foram formuladas para conter $150 \mathrm{mg}$ Fe/kg de ração.
Tabela 2. Concentração de ferro nas rações de repleção de leitões anêmicos. Média, desvio-padrão e coeficiente de variação (\%). Determinações em triplicata.

\begin{tabular}{|c|c|c|c|c|}
\hline Grupo & $n$ & Fonte de Ferro & $\mathrm{mgFe} / \mathrm{kg}$ & C.V.\% \\
\hline Controle & 5 & $\mathrm{FeSO}_{4} \cdot 7 \mathrm{H}_{2} \mathrm{O}$ & $141,4(38,3)^{\mathbf{a}}$ & 27 \\
\hline Ferlim & 5 & $\mathrm{FeSO}_{4}$ microencapsulado & $150,5(6,1)^{a}$ & 4 \\
\hline $\mathrm{Fe}^{0}$ & 5 & Fe reduzido eletrolitícamente & $143,5(11,4)^{a}$ & 8 \\
\hline
\end{tabular}

Sobrescritos diferentes correspondem a resultados significantemente diferentes $(p<0,05)$.

Repleção de hemoglobina: Os animais anêmicos foram agrupados aleatoriamente em 3 grupos com base no pool de ferro dos animais, obtido pelo produto peso $(\mathrm{kg}) \times \mathrm{Hb}(\mathrm{g} / \mathrm{L})$, e receberam rações com diferentes fontes de ferro ou concentração por 13 dias. Durante esse período, o consumo de ração foi medido diariamente. Pesagem e determinação da hemoglobina foram efetuadas nos dias 0, 6 e 13. Rações e água foram oferecidas ad libitum. O cálculo do pool de ferro para leitões foi feito segundo a equação $(1)^{26,27}$. Assumiu-se um volume de sangue igual a $6 \%$ do peso corporal do animal.

Pool de Fe $(\mathrm{mg})=\mathrm{P}(\mathrm{kg}) \times \mathrm{Hb}(\mathrm{g} / \mathrm{L}) \times 0,06$ $(\mathrm{L} / \mathrm{kg}) \times 3,35(\mathrm{mgFe} / \mathrm{gHb}) \quad(1)$

O cálculo da biodisponibilidade de ferro (\% $A B S$ ) foi feito a partir da variação do poo/ de ferro (pool final - pool inicial) e do ferro ingerido pelos animais, para cada fonte de ferro testada (Equação 2).

$\% \mathrm{ABS}=\frac{\text { Pool de ferro final }(\mathrm{mg})-\text { Pool de ferro inicial }(\mathrm{mg})}{\text { Fe total ingerido }(\mathrm{mg})}$

Valor Biológico Relativo (RBV): O Valor Biológico Relativo ao $\mathrm{FeSO}_{4} \cdot 7 \mathrm{H}_{2} \mathrm{O}$ foi calculado como a porcentagem do ferro absorvido relativa à absorção do ferro da dieta controle contendo sulfato ferroso (equação 3).

RBV $=100$ (\% Fe ABS do grupo experimental/\% Fe ABS do grupo padrão)

(3)

Determinação do ferro dialisável: O ferro dialisável foi determinado em leite em pó reconstituído, de acordo com o método proposto por Miller ${ }^{28}$. Os compostos foram adicionados ao 
leite reconstituído (16mgFe/L) e , após acidificação com $\mathrm{HCl}$ até $\mathrm{pH} 2$ (Merck ${ }^{\circledR}$ p.a.), alíquotas de $20 \mathrm{~g}$ em triplicata foram submetidas à digestão com pepsina suína $\left(\right.$ Sigma $\left.^{\circledR}\right)$. Em seguida, foram adicionados tubos de diálise (Spectra/Por ${ }^{\circledR}$ Membrane com largura do poro de $6-8 \mu \mathrm{m}$ ) contendo solução de $\mathrm{NaHCO}_{3}$ (Merck ${ }^{\circledR}$ p.a.) para alcalinização do meio. Ao término dessa digestão, a amostra foi submetida a uma segunda digestão com uma solução de pancreatina-bile suína $\left(\right.$ Sigma $\left.^{\circledR}\right)$. Após o último período de digestão, os segmentos de tubos de diálise foram lavados e seu conteúdo, contendo o ferro dialisado, foi diluído para $25 \mathrm{~mL}$ com água deionizada. O ferro dialisável (\%FeD) foi expresso em porcentagem do Fe total presente na amostra (Tabela 3), de acordo com a equação (4):

$\% \mathrm{FeD}_{\text {dialisável }}=100 \times \frac{\mu \mathrm{g} \mathrm{Fe} / \mathrm{mL} \text { dialisado } \times \mathrm{mL} \text { dialisado }}{\mu \mathrm{g} \mathrm{Fe} / \mathrm{g} \text { amostra } \times 20 \mathrm{~g}}$

Concentração de ferro nas rações: A concentração de ferro nas amostras foi determinada, após digestão das amostras com $\mathrm{HNO}_{3}: \mathrm{HClO}_{4}(5: 1)$, pelo método de Espectrofotometria de Absorção Atômica (EAA), segundo as normas da AOAC ${ }^{29}$ (Tabela 2). Uma curva padrão de ferro foi preparada com padrão de Cloreto Férrico (9972 Titrisol - Merck ${ }^{\circledR}$ ) contendo 1.000mg de $\mathrm{Fe} / \mathrm{L}$ diluído em água deionizada no intervalo de 0,3 a 5,0 $\mu \mathrm{g} \mathrm{Fe} / \mathrm{mL}$. A absorbância das soluções foi determinada em EAA Hitachi Z5000, com lâmpada de catodo oco, calibrado nas seguintes condições: região ultravioleta, comprimento de onda $248,3 \mathrm{~nm}$, fenda $0,2 \mathrm{~nm}$ e chama oxidante de ar/acetileno.

Ferro total no fígado: Ao término do ensaio, os animais, após insensibilização elétrica, foram sacrificados para retirada do fígado. Cada fígado foi acondicionado em saco plástico identificado com etiqueta e congelado a $-20^{\circ} \mathrm{C}$ até o momento da análise. Os fígados foram descongelados à temperatura ambiente para a determinação do ferro total. Aproximadamente $1 \mathrm{~g}$ de cada fígado, retirado do $4^{\circ}$ quadrante do lobo direito, foi digerido com $\mathrm{HNO}_{3}\left(\right.$ Merck $^{\circledR}$ p.a.). A concentração de ferro nas amostras foi determinada pelo método de Espectrofotometria de Absorção Atômica (EAA), segundo as normas da AOAC ${ }^{29}$.

Ferro heme no fígado: A determinação do ferro heme no fígado foi feita após extração da hematina com acetona, seguindo o procedimento descrito por Hornsey ${ }^{30}$ para análise de pigmentos totais. Utilizou-se hemoglobina bovina liofilizada H-2500 $\left(\right.$ Sigma $\left.^{\circledR}\right)$ como padrão de referência secundário. A extração de pigmentos totais foi realizada adicionando-se às amostras $40 \mathrm{~mL}$ de acetona (Merck ${ }^{\circledast}$ p.a.), $1 \mathrm{~mL}$ de solução de L-cisteína $1 \%, 1 \mathrm{~mL}$ de ácido clorídrico concentrado (Merck ${ }^{\circledR}$ p.a.) e água suficiente para atingir um volume de $50 \mathrm{~mL}$, considerando-se a umidade da amostra. O extrato foi filtrado e a leitura foi feita em espectrofotômetro HITACHI ${ }^{\circledR}$ (Mod. U-34410), em comprimento de onda de $640 \mathrm{~nm}$. A absorbância encontrada foi multiplicada pelo fator 6800, obtido a partir do coeficiente de extinção molar da hematina e das diluições feitas pelo coeficiente de extinção molar, e dividido pelo peso da amostra, obtendo-se a concentração de hematina em $\mu \mathrm{g} / \mathrm{g}$ de amostra. A concentração de ferro heme foi calculada considerando-se que a hematina possui $8,82 \%$ de ferro ${ }^{30}$.

Ferro não-heme no fígado: O ferro não-heme do fígado foi calculado subtraindo-se o ferro heme do ferro total. Este corresponde ao ferro de reserva (ferritina).

Análise estatística: Para verificar a existência de diferenças estatisticamente significantes entre os grupos utilizou-se a Análise de Variância (ANOVA). Para avaliar diferenças significantes na concentração de ferro das rações, utilizou-se o método das Comparações Múltiplas de Tamhane. Nas duas avaliações, o nível de significância estabelecido foi de $5 \%$. O programa utilizado foi SPSS for Windows version 11.5.

\section{RESULTADOSE DISCUSS ÃO}

A microencapsulação de sulfato ferroso desenvolvida pelo Laboratório de Tecnologia de Partículas do IPT, em parceria com o Laboratório 
Tabela 3. Porcentagem de ferro dialisado (\%FeD) em leite em pó reconstituído (16mg Fe/L). Média e desvio-padrão $(n=3)$.

\begin{tabular}{lc}
\hline Composto & $\% \mathrm{FeD}$ \\
\hline $\mathrm{FeSO}_{4} \cdot 7 \mathrm{H}_{2} \mathrm{O}$ & $2,2(0,1)^{\mathbf{a}}$ \\
$\mathrm{Ferlim}^{1}$ & $3,4(0,1)^{\mathbf{b}}$ \\
$\mathrm{Fe}^{\mathbf{2}}$ & $3,6(0,0)^{\mathbf{b}}$ \\
\hline
\end{tabular}

Sobrescritos diferentes correspondem a resultados significantemente diferentes $(p<0,05)$; ${ }^{1}$ Ferlim: Sulfato ferroso microencapsulado; ${ }^{2}$ Ferro eletroliticamente reduzido.

de Nutrição do Departamento de Alimentos e Nutrição Experimental da FCF/USP/SP, teve como objetivo produzir um composto que não interagisse com o meio ao qual foi adicionado, mas que fosse biodisponível. Dentre várias formulações desenvolvidas e testadas, selecionou-se o composto microencapsulado denominado Ferlim, que apresentou 3,4, DP=0,1\% de ferro dialisado, enquanto o $\mathrm{FeSO}_{4}$ apresentou 2,2, DP=0,1\%. Para - $\mathrm{Fe}^{0}$, a porcentagem de diálise foi de 3,6, DP $=0,0 \%$ (Tabela 3). O menor valor obtido para o $\mathrm{FeSO}_{4}$ não encapsulado, provavelmente, ocorreu pela oxidação desse composto, o que mostra a importância da sua microencapsulação. Com relação ao $\mathrm{Fe}^{0}$, a porcentagem de $3,6 \%$ de diálise é coerente com a característica das partículas utilizadas, já que $98 \%$ das mesmas tinham tamanho inferior a $40 \mu \mathrm{m}$. No $\mathrm{pH} 2$ a que a amostra é submetida na simulação da digestão gástrica, a maior parte já é oxidada a $\mathrm{Fe}^{2+}$, tornando-se altamente solúvel e, portanto, com boa dialisabilidade. Vale lembrar que o ensaio in vitro, por ser realizado em uma dieta pontual e por não sofrer a influência de fatores fisiológicos que podem afetar a biodisponibilidade do ferro, prediz a tendência mas não a magnitude da resposta absortiva observada in vivo.

A biodisponibilidade dos compostos de ferro avaliada neste ensaio, expressa pelas porcentagens de absorção e de absorção relativa ao $\mathrm{FeSO}_{4} \cdot 7 \mathrm{H}_{2} \mathrm{O}$, não foi diferente $(p>0,05)$. A biodisponibilidade do Ferlim foi de $15,0 \%$ e a do $\mathrm{Fe}^{0}$ de $12,8 \%$ (Tabela 4). Segundo Monsen ${ }^{31}$, quando os estoques de ferro corporal estão depletados, são esperados valores de absorção entre $10.0 \%$ e $20,0 \%$ em refeições com média e elevada biodisponibilidade de ferro, respectivamente. Dessa forma, os resultados de absorção acima descritos indicam que os dois compostos testados apresentaram biodisponibilidade equivalente àquela obtida com refeições com média biodisponibilidade de ferro.

Tabela 4. Parâmetros de recuperação de hemoglobina em leitões anêmicos, alimentados por 13 dias com rações com diferentes compostos de ferro. Média e desvio-padrão.

\begin{tabular}{|c|c|c|c|c|c|c|c|}
\hline \multirow{2}{*}{ Parâmetros biológicos } & \multirow{2}{*}{ Dia } & \multicolumn{2}{|c|}{${ }^{1}$ Controle $(n=7)$} & \multicolumn{2}{|c|}{${ }^{2}$ Ferlim $(n=8)$} & \multicolumn{2}{|c|}{${ }^{3} \mathrm{Fe}^{0}(\mathrm{n}=8)$} \\
\hline & & M & DP & M & DP & $M$ & $\mathrm{DP}$ \\
\hline \multirow[t]{3}{*}{ Peso (kg) } & 0 & 8,1 & $1,2^{\mathrm{a}}$ & 6,3 & $1,5^{a}$ & 6,7 & $1,4^{\mathrm{a}}$ \\
\hline & 6 & 10,0 & $1,6^{\mathbf{b}}$ & 8,2 & $1,9^{b}$ & 8,5 & $2,1^{b}$ \\
\hline & 13 & 11,6 & $1,7^{c}$ & 10,7 & $2,3^{c}$ & 10,9 & $3,3^{c}$ \\
\hline \multirow[t]{3}{*}{ Hemoglobina (g/L) } & 0 & 60,3 & $10,7^{\mathrm{a}}$ & 72,5 & $12,8^{a}$ & 69,0 & $13,5^{\mathrm{a}}$ \\
\hline & 6 & 81,1 & $12,2^{\mathbf{b}}$ & 82,4 & $13,6^{b}$ & 81,6 & $12,0^{\mathbf{b}}$ \\
\hline & 13 & 100,9 & $10,0^{c}$ & 96,8 & $8,2^{c}$ & 93,9 & $13,8^{c}$ \\
\hline \multirow[t]{3}{*}{ Fe POOL (mg) } & 0 & 99,1 & $23,3^{a}$ & 91,2 & $22,7^{a}$ & 91,7 & $23,4^{a}$ \\
\hline & 6 & 165,2 & $44,3^{b}$ & 137,1 & $43,0^{b}$ & 139,4 & $44,5^{b}$ \\
\hline & 13 & 236,9 & $49,7^{c}$ & 212,1 & $74,6^{c}$ & 209,0 & $78,3^{c}$ \\
\hline Fe ingerido (mg) & 13 & 861,8 & $159,4^{a}$ & 783,9 & $249,8^{a}$ & 848,5 & $356,9^{a}$ \\
\hline Absorção (\%) & & 16,0 & $3,1^{\mathrm{a}}$ & 15,1 & $3,8^{a}$ & 12,8 & $4,3^{\mathrm{a}}$ \\
\hline Absorção Relativa (\%) & & & & 94,2 & $23,8^{a}$ & 79,7 & $26,6^{\mathrm{a}}$ \\
\hline
\end{tabular}

a,b,c Sobrescritos diferentes na mesma linha correspondem a resultados significantemente diferentes $(p<0,05)$; ${ }^{1}$ Controle (ração fortificada com $\mathrm{FeSO}_{4} \cdot 7 \mathrm{H}_{2} \mathrm{O}$ na concentração de 141,4mgFe/kg ração); ${ }^{2} \mathrm{Ferlim}$ (ração fortificada com FeSO ${ }_{4}$ microencapsulado com alginato na concentração de $150,5 \mathrm{mgFe} / \mathrm{kg}$ ração); ${ }^{3} \mathrm{Fe}^{0}$ (ração fortificada com ferro eletrolítico na concentração de $143,5 \mathrm{mgFe} / \mathrm{kg}$ ração). 
O valor biológico relativo (RBV) para o grupo Ferlim foi de 94,2\%, o que denota a similaridade entre este e $\mathrm{O} \mathrm{FeSO}_{4}$ não encapsulado. No caso do grupo $\mathrm{Fe}^{0}$, a RBV foi de 79,7\%, resultado semelhante aos obtidos por Forbes et al. ${ }^{10}$, tanto pelo método da AOAC modificado como pelo método da eficiência de regeneração da hemoglobina (HRE) em ratos anêmicos, utilizando rações à base de caseína. Apesar de não diferirem estatisticamente, há uma clara tendência de o grupo $\mathrm{Fe}^{0}$ apresentar menor RBV. Deve-se salientar que, para ser absorvido, o $\mathrm{Fe}^{0}$ deve ser, primeiramente, oxidado a $\mathrm{Fe}^{+2} \mathrm{ou} \mathrm{Fe}^{+3}$, o que ocorre no pH ácido do estômago e como foi observado no método in vitro. Entretanto, segundo Moughan ${ }^{24}$, em leitões com quatro semanas de vida, como os utilizados neste ensaio, o pH gástrico, como em bebês humanos, é em torno de 5,0 o que pode ter levado à menor oxidação do $\mathrm{Fe}^{0}$, explicando a tendência à menor porcentagem de absorção observada.

A microencapsulação de sulfato ferroso já havia se mostrado como boa alternativa para proteger os alimentos de alterações organolépticas indesejáveis e proteção do sulfato ferroso à oxidação. Diversos trabalhos realizados com o composto de ferro microencapsulado com lecitina de soja, SFE-171 ${ }^{\oplus}$, já comprovavam a microencapsulação do sulfato ferroso como importante alternativa para a utilização do mesmo. Os resultados de absorção e de absorção relativa obtidos neste ensaio corroboram os obtidos com o SFE-171 ${ }^{\circledR 14-17}$. Uma constatação interessante foi que o Ferlim distribuiu-se muito homogeneamente na ração, o que resultou em um coeficiente de variação de $4 \%$ na análise de ferro total, contras- tando com os de $27 \%$ do $\mathrm{FeSO}_{4} \cdot 7 \mathrm{H}_{2} \mathrm{O}$ e $8 \%$ do $\mathrm{Fe}^{0}$ (Tabela 2). Esse dado deve ser considerado quando se pensar, por exemplo, na fortificação de farinhas ou mesmo na produção de rações para animais.

No fígado (Tabela 5), um compartimento de reserva corporal de ferro, não houve diferença entre todos os grupos experimentais quanto ao peso do órgão, ferro total, ferro heme e ferro não-heme $(p>0,05)$. Todavia, houve uma clara tendência a menor concentração de ferro heme $[10,5$ $(2,5) \mu \mathrm{g} / \mathrm{g}]$ e ferro não-heme $[28,6(22,3) \mu \mathrm{g} / \mathrm{g}]$ no fígado dos leitões que receberam rações fortificadas com Fe $\mathrm{Fe}^{0}$. Para o grupo Ferlim estas concen-

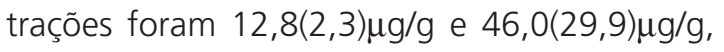
respectivamente, indicando a menor biodisponibilidade do $\mathrm{Fe}^{0}$.

Sabe-se que o ferro não-heme no fígado está na forma de ferritina e hemossiderina e que estes representam, aproximadamente, $80 \%$ do ferro total do órgão ${ }^{32}$. Os resultados do presente trabalho confirmam estes dados. A análise de ferro no fígado demonstrou que, do ferro total, $78 \%$ (Ferlim) e $73 \%$ ( $\left.\mathrm{Fe}^{0}\right)$ estavam na forma de ferro não-heme. Entretanto, cabe salientar que o grupo que recebeu Ferlim apresentou tendência, não significante $(p>0,05)$, à maior concentração de ferro não-heme no fígado (similar ao observado para os grupos que receberam $\mathrm{FeSO}_{4} \cdot 7 \mathrm{H}_{2} \mathrm{O}$ ), do que o grupo que recebeu $\mathrm{Fe}^{0}$. Este resultado torna-se de grande importância, considerando que a fortificação de alimentos com sais de ferro é indicada para as populações com elevada prevalência de anemia por deficiência de ferro. Dessa forma, compostos que, além de elevar a concentração sanguínea de hemoglobina, também elevem os estoques de ferro corporal, como o Ferlim,

Tabela 5. Peso do fígado e concentração de ferro heme e ferro não-heme no fígado dos leitões.

\begin{tabular}{|c|c|c|c|c|c|c|c|c|c|}
\hline \multirow{2}{*}{ Grupo } & \multirow{2}{*}{$n$} & \multicolumn{2}{|c|}{ Peso fígado $(\mathrm{g})$} & \multicolumn{2}{|c|}{ Ferro total $(\mu \mathrm{g} / \mathrm{g})$} & \multicolumn{2}{|c|}{ Ferro heme $(\mu \mathrm{g} / \mathrm{g})$} & \multicolumn{2}{|c|}{ Ferro não-heme $(\mu \mathrm{g} / \mathrm{g})$} \\
\hline & & M & $\mathrm{DP}$ & M & DP & M & DP & M & $\mathrm{DP}$ \\
\hline Controle & 7 & 263,4 & $50,0^{a}$ & 67,4 & $18,1^{b}$ & 15,4 & $5,3^{c}$ & 53,7 & $22,0^{d}$ \\
\hline Ferlim & 8 & 236,4 & $53,3^{a}$ & 58,8 & $30,9^{b}$ & 12,8 & $2,3^{c}$ & 46,0 & $29,9^{d}$ \\
\hline $\mathrm{Fe}^{0}$ & 8 & 252,5 & $76,3^{a}$ & 39,2 & 23,2 & 10,5 & $2,5^{c}$ & 28,6 & $22,3^{d}$ \\
\hline
\end{tabular}

a,b,c,d Sobrescritos diferentes na mesma coluna correspondem a resultados significantemente diferentes $(p<0,05)$. 
podem contribuir para a manutenção dos níveis adequados de hemoglobina por um maior período de tempo.

Os resultados obtidos demonstraram que a microencapsulação de sulfato ferroso (Ferlim) não diminuiu a biodisponibilidade do $\mathrm{FeSO}_{4}$. Testes prévios de dissolução in vitro mostraram que sulfato ferroso microencapsulado possui elevada solubilidade, rapidamente liberando o sulfato ferroso ${ }^{21}$. Por outro lado, vale salientar que os valores médios de porcentagem de absorção, de RBV, de concentrações de ferro total e ferro não-heme no fígado dos leitões, foram menores no grupo que recebeu $\mathrm{Fe}^{0}$. Essa tendência, não confirmada estatisticamente, em função da grande dispersão dos resultados intragrupos, merece ser reavaliada, aumentando-se o número de animais por grupo.

\section{O N CLUS Ã O}

O objetivo da microencapsulação é proteger o sulfato ferroso de oxidação processo que, além de diminuir sua biodisponibilidade, provoca alterações organolépticas indesejáveis nos alimentos. Os resultados obtidos neste trabalho demonstraram que a microencapsulação do sulfato ferroso não alterou a sua biodisponibilidade, apontando a utilização dessa nova forma microencapsulada de sulfato ferroso (Ferlim) como alternativa tecnológica para viabilizar a utilização do mesmo na fortificação de alimentos desidratados.

\section{A GRADECIMENTOS}

Ao Conselho Nacional de Desenvolvimento Científico e Tecnológico (CNPq), pela concessão da Bolsa de Mestrado (processo no 130.548/2002-2 ) e à empresa Fermavi Eletroquímica Ltda, pelo apoio financeiro.

\section{REFERÊ N CIAS}

1. Brasil. Agência Nacional de Vigilância Sanitária. Resolução RDC n.344, de 13 de dezembro de
2002. Legislação Específica por Área de Assunto. Regulamentos técnicos por assunto: F. Farinha de trigo e/ou milho fortificadas com ferro [acesso em 22 out 2003]. Disponível em: http://e-legis. bvs.br/ leisref/public/showAct. php?id=1679

2. Salzano AC, Torres MAA, Batista Filho M, Romani SAM. Anemia em crianças de dois serviços de saúde de Recife, PE (Brasil). Rev Saúde Pública. 1985; 19(6):499-507.

3. Cardoso MA, Ferreira MV, Camargo LMA, Szarfarc S. Anemia em população de área endêmica de malária, Rondônia (Brasil). Rev Saúde Pública. 1992; 26(3):161-6.

4. Soares NT, Guimarães ARP, Sampaio HAC, Almeida PC, Coelho RR. Estado nutricional de lactentes em áreas periféricas de Fortaleza. Rev Nutr. 2000; 13(2):99-106.

5. Alberico APM, Veiga GV, Baiao MR, Santos MMAS, Souza SB, Szarfarc SC. Iron deficiency anaemia in infants attended at municipal primary health care centres in Rio de Janeiro - Brazil. Nutr Food Sci. 2003; 33(2):50-5.

6. Björn-Rasmussen E, Hallberg L, Rossander L. Absorption of fortification iron. Br J Nutr. 1977; 37(3):375-88.

7. Cook JD, Reusser ME. Iron fortification: an update. Am J Clin Nutr. 1983; 38(4):648-59.

8. Yip R. Prevention and control of iron deficiency: policy and strategy issues. J Nutr. 2002; 132: 802S-5S.

9. Waddell J. Bioavailability of iron sources. Food Prod Dev. 1974; 8(2):80-6.

10. Forbes AL, Adams CE, Arnaud MJ, Chichester CO, Cook JD, Harrison BN, et al. Comparison of in vitro, animal, and clinical determinations of iron bioavailability: International Nutritional. Anemia Consultative Group Task Force report on iron bioavailability. Am J Clin Nutr. 1989; 49(2): 225-38.

11. Jackson LS, Lee K. Microencapsulated iron for food fortification. J Food Sci. 1991; 4:1047-51.

12. Lee K, Clydesdale FM. Iron sources in food fortification and their changes due to food processing. Crit Rev Food Sci Nutr. 1978; 11(2): 117-53.

13. Boccio JR, Zubillaga MB, Caro RA, Gotelli CA, Gotelli MJ, Weill R. A new procedure to fortify fluid milk and dairy products with high bioavailable ferrous sulfate. Nutr Rev. 1997; 55(6):240-6.

14. Gotelli CA, Gotelli MJ, Boccio JR, Zubillaga MB, Caro RA, Garcia Del Rio H, et al. Bioavailability of microencapsulated ferrous sulfate in fluid milk studies in human beings. Acta Physiol Pharmacol Ther Latinoam. 1996; 46(4):239-45. 
15.Zubillaga MB, Caro RA, Boccio JR, Gotelli CA, Gotelli MJ, Weill R. New procedure to fortify fluid milk with iron: metabolic and biochemical study in rats. Nutr Res. 1996; 16(1):131-7.

16. Boccio JR, Zubillaga MB, Caro RA, Gotelli CA, Gotelli MJ, Weill R. Bioavailability and stability of microencapsulated ferrous sulfate in fluid milk: studies in mice. J Nutr Sci Vitaminol. 1996; 42(3): 233-9.

17. Lysionek $A E$, Zubillaga $M B$, Salgueiro MJ, Pineiro A, Caro RA, Weill, et al. Bioavailability of microencapsulated ferrous sulfate in powdered milk produced from fortified fluid milk: a prophylactic study in rats. Nutrition. 2002; 18(3): 279-81.

18. Ré MI, Fernandes FC. Produto à base de sulfato ferroso para fortificação de alimentos desidratados e método de preparação. Brasil patente. PI 005819. 24 nov 2003.

19. Ré MI, Fernandes FC. A ferrous sulphate-based product to be used for fortifying dehydrated food stuff and method for the preparation thereof'. Brasil patente. PCT/BR2004/000231. 23 nov 2004.

20. Ré MI, Fernandes FC. Produto à base de sulfato ferroso para fortificação de alimentos desidratados e método de preparação. Brasil patente. PI 0305871-9.

21. Ré MI. Desenvolvimento de micropartículas de sulfato ferroso modificadas para uso em estudo clínico de combate à anemia ferropriva em pré-escolares. Relatório técnico. São Paulo: Instituto de Pesquisas Tecnológicas da Universidade de São Paulo. Fapesp; 2003. Processo n.67459.

22. Perks SM, Miller DD. Adding ascorbic acid to ironfortified cow's milk does not enhance iron bioavailability to piglets. Nutr Res. 1996; 16: 969-75.
23. Miller ER, Ullrey DE. The pig as a model for human nutrition. Ann Rev Nutr. 1987; 7:361-82.

24. Moughan PJ, Birtles MJ, Cranwell PD, Smith WC, Pedraza M. The piglet as a model animal for studying aspects of digestion and absorption in milk-fed human infants. World Rev Nutr Diet. 1992; 67:40-113.

25. National Research Council. In: Nutrient requirements of swine: subcommittee on swine nutrition. 10th ed. Washington (DC): National Academy Press; 1998.

26. Miller J. Assessment of dietary iron availability by rat hemoglobin repletion assay. Nutr Rep Int. 1982; 26(6):993-1005.

27. South PK, Lei X, Miller DD. Meat enhances nonheme iron absorption in pigs. Nutr Res. 2000; 20(12):1749-59.

28. Miller DD, Schricker RR, Rasmussen RR, Van Campen D. An in vitro method for estimation of iron availability from meals. Am J Clin Nutr. 1981; 34:2248-56.

29. Association Official Analytical Chemists. Official methods of analysis. 13th ed. Arlington (VA): AOAC; 1985. p.505.

30. Hornsey HC. Estimation of the nitric oxid-haem pigments. J Sci Food Agric. 1956; 7:534-40.

31. Monsen ER, Hallberg L, Layrisse M, Hegsted M, Cook JD, Mertz W, et al. Estimation of available dietary iron. Am J Clin Nutr. 1978; 31:134-141.

32. Hazell T. Mineral in foods: dietary sources, chemical forms, interaction, biovailability. World Rev Nutr Diet. 1992; 67:40-113.

Recebido em: 25/8/2006

Versão final reapresentada em: 21/11/2006 Aprovado em: 11/12/2006 
